\title{
E-Beam Destruction of Contaminants in Water
}

\section{Summary}

Next-generation electron beam (E-beam) technology creates the potential to treat the complex and difficult waste streams of today and tomorrow. Fermi National Accelerator Laboratory brings 50 years of groundbreaking E-beam experience across technology research, innovation, and application to unlocking this potential from a capacity and cost perspective using proprietary Lab intellectual property. Fermilab's technology will deliver a high throughput, portable, scalable, and cost-effective solution to a wide variety of contaminants. Potential applications include contaminants of increasing health, regulatory, economic, and public relations concerns.

\section{Key Benefits}

Fermilab waste "Swiss Army Knife" treats multiple contaminants simultaneously, including:
Perfluorates
PCBs
TCE

Pharmaceuticals

MTBE

Pathogens

Dyes

Organics

Agricultural run off

\section{- Decreased susceptibility to additional} contaminants - such as organic compounds and dissolved solids - that significantly reduce the efficacy of existing and near-term solutions

Key Fermilab improvements over existing E-beam technology include:

- Increased device power enables 500,000 gallons/day throughput per unit

- New cooling approach reduces size from a three-story building to a compact and portable skid-mounted unit

- Superconducting technology increases energy efficiency by $50 \%$ and decrease overall OpEx costs by $30 \%$

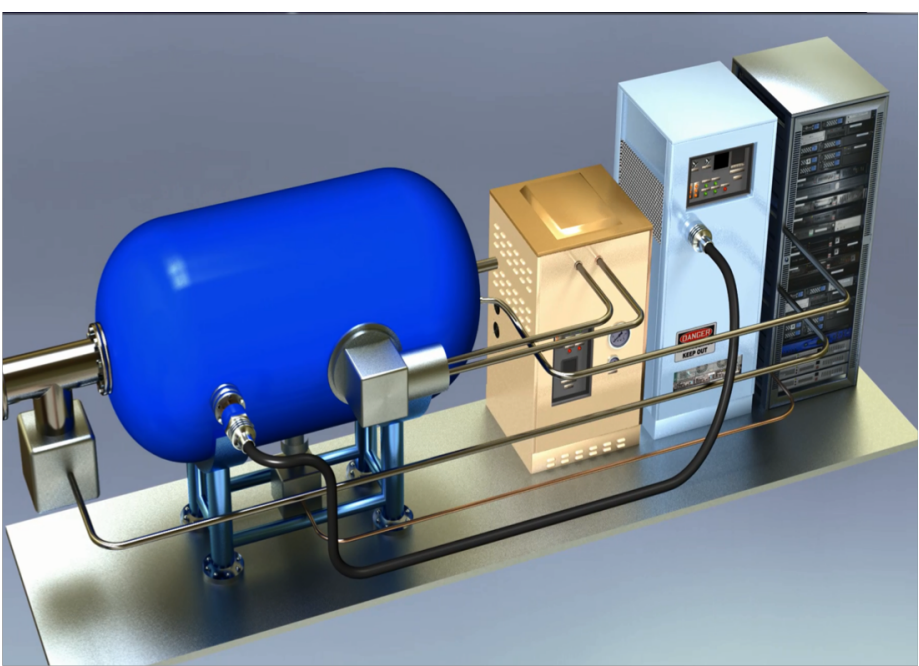

High Power and Energy Efficient Electron Beam Accelerator Being Developed at Fermilab.

\section{Future Benefits}

Technology mitigates future treatment, cost, compliance, and risk issues:

- Avoids the long ramp up period to develop new solutions for the next high-profile contaminant - and the next, and the next given its broad-acting treatment approach

- Avoids the capital, operational, and space costs of deploying parallel treatment technologies for many existing and new contaminants

\section{Technology Roadmap}

- Laboratory validation of E-beam treatment of single contaminants

- Field sample testing of complex wastewater streams

- Field engineering study to define key device specifications and requirements

- Development and testing of $20 \%$ field demonstration unit

- Manufacturing of full-scale unit

Charlie Cooper ccooper@fnal.gov http://iarc.fnal.gov/
This manuscript has been authored by Fermi Research Alliance, LLC under Contract No. DE-AC02-07CH11359 with the U.S. Department of Energy, Office of Science, Office of High Energy Physics 